\title{
Bar-Halo Interaction and Bar Growth
}

\author{
E. Athanassoula \\ Observatoire de Marseille, 2 Place Le Verrier, 13248 Marseille cedex 04, France
}

\begin{abstract}
I show that strong bars can grow in galactic discs, even when the latter are immersed in haloes whose mass within the disc radius is comparable to, or larger than, the mass of the disc. I argue that this is due to the response of the halo and in particular to the destabilising influence of the halo resonant stars. Via this instability mechanism the halo can stimulate, rather than restrain, the growth of the bar.
\end{abstract}

Subject headings: galaxies: evolution, galaxies: halos, galaxies: kinematics and dynamics

\section{Introduction}

Galactic discs are generally unstable and form bars within a few dynamical timescales (e.g. Athanassoula 1984, or Sellwood \& Wilkinson 1993, for reviews). The first remedy proposed against this instability is to immerse the disc in a massive halo component. Ostriker \& Peebles (1973), from an insightful analysis of $N$-body simulations with a very small number of particles, were the first to outline the stabilising influence of haloes. They further suggested that halo-to-disc mass ratios of 1 to 2.5 (interior to the disc) are required for stability. Athanassoula \& Sellwood (1986) measured the growth rates of the most unstable bar-forming modes in 2D $N$-body simulations and showed that the growth rate is smaller in cases with large halo-to-disc mass ratios. Unfortunately, no such analysis has been made for fully selfconsistent 3D N-body simulations. On the other hand, Athanassoula \& Misiriotis (2002, hereafter AM) and Athanassoula (2002a,b) argue that stronger bars can grow in simulations with initially very high halo-to-disc mass ratios than in simulations with lower halo-to-disc mass ratios. In order to understand whether the above pieces of evidence are contradictory, and, if so, to attain a coherent picture, I will examine in this letter the role of the halo in the formation of the bar. Several papers have focused on the influence of the halo on the slowing down of the bar (e.g. Tremaine \& Weinberg 1984, Weinberg 1985, Combes et al. 1990, Little \& Carlberg 1991, Hernquist \& Weinberg 1992, Athanassoula 1996, Debattista \& Sellwood 
1998 and 2000). This paper is complementary, since it concentrates on the role of the halo in determining the bar growth. The discussion will focus on three simulations. The results, however, are based on a much larger set of simulations, leading to the same conclusions.

\section{Results on bar formation}

I will describe the results of three fully self-consistent 3D $N$-body simulations of isolated galaxies consisting of a disc and a halo component. The initial conditions and the numerical methods are described in AM, whose notation I follow here. The three initial conditions have identical exponential discs of unit mass and scale length, scale height $z_{0}=0.2$ and $Q$ $=1$. Their halos are five times as massive as their discs and have different degrees of central concentration. They are initially isotropic, spherical and non-rotating, with a radial density profile

$$
\rho_{h}(r)=\frac{M_{h}}{2 \pi^{3 / 2}} \quad \frac{\alpha}{r_{c}} \frac{\exp \left(-r^{2} / r_{c}^{2}\right)}{r^{2}+\gamma^{2}}
$$

with $M_{h}=5, r_{c}=10$. The parameter $\alpha$ is a normalisation constant defined by

$$
\alpha=\left[1-\sqrt{\pi} \exp \left(q^{2}\right)(1-\operatorname{erf}(q))\right]^{-1}
$$

where $q=\gamma / r_{c}$ and $\operatorname{erf}$ is the error function (Hernquist 1993). The initial circular velocity curves of the three models are shown in the upper panels of Fig. 1. The first simulation, hereafter $\mathrm{MD}$, has $\gamma=5$, and its disc dominates in the inner parts, roughly up to $r=5$. In the other two (hereafter $\mathrm{MH}$ and $\mathrm{RH}$, respectively), $\gamma=0.5$, and the halo contribution is slightly larger than that of the disc up to the maximum of the disc rotation curve, and considerably so at larger radii. Model $\mathrm{RH}$ is identical to model $\mathrm{MH}$, except that its halo is rigid - i.e. given by a potential imposed on the disc particles - and does not evolve during the simulation. The number of particles in the simulations is 1131206, 1163030 and 200000, respectively. The simulations were run on a GRAPE-5 system with a softening of 0.0625 and a time-step of 0.015625. I assessed the numerical robustness of my results by trying double the number of particles, different values of the softening and time step, as well as direct summation and a non-GRAPE tree code. For a reasonable calibration (AM), $t=500$ corresponds to $7 \times 10^{9}$ years. Different values, however, can be obtained for different scalings of the disc mass and scale length.

The second row of panels of Fig. 1 gives the three circular velocity curves at time $t=800$. Note that the disc material has moved considerably inwards in the two first cases, but very 
little in the third one. In model MD the difference between times 0 and 800 is quantitative, since the disc dominates the inner parts both initially and after the evolution, albeit to a larger degree after the bar has grown. On the other hand for model MH the difference is qualitative, since at $t=800$ the inner regions are dominated by the disc, contrary to what was the case initially. The third row of panels gives the face-on view of the disc component. The differences between the properties of the three models are striking. A comparison of models $\mathrm{MD}$ and $\mathrm{MH}$ shows that the bar that grew in the initially more halo-dominated environment is stronger than the bar that grew in the disc-dominated environment. It is longer and its isophotes are more rectangular-like. The strength of the bar can be measured with the help of the relative amplitude of the Fourier components of the density or mass (AM), and the last row of panels in Fig. 1 shows that indeed the $\mathrm{MH}$ bar is considerably stronger than the MD one. The $m=4,6$ and 8 Fourier components of the face-on density of model MH are also considerably stronger than those of model MD (AM).

Very strong differences are also found when comparing models $\mathrm{MH}$ and $\mathrm{RH}$. Model MH has a very strong bar, while model RH has a very mild oval in the innermost parts. Their edge-on views are also very different (not shown here, cf. Athanassoula 2002b for another example). Model MH seen side-on has a strong peanut or ' $\mathrm{X}$ '-shape, and a big bulge-like protuberance if seen end-on. Model RH shows no such features. Finally the difference between their integrated Fourier components is striking.

The three models differ also in the way their bars evolve. In model MD the bar grows very rapidly during the first part of the evolution, but little, if at all, after that. On the contrary the growth of the bar in model MH during the first part of the evolution is slower than in MD, but stays considerable, although less important than in the first part, till the end of the simulation. The pattern speed of model MH starts off higher than that of model MD, but ends smaller. The pattern speed of MD also decreases with time, but less so (cf. Debattista \& Sellwood 1998, 2000). The pattern speed of the non-axisymmetric component in RH can not be measured reliably before $t=300$, and after that does not show any signs of decrease. For models MD and MH there is exchange of energy and angular momentum between the disc and halo components, so that the halo, which was initially non-rotating, displays rotation after the bar has grown. This is small for model MD and considerable for model $\mathrm{MH}$, for which at $t=900$ the halo has somewhat less than a half of the angular momentum of the disc, i.e. not far from a third of the total. 


\section{The role of the halo}

In order to understand the role of the halo in the formation and evolution of the bar, I froze the potential at four selected times during each simulation and chose randomly 100000 disc and 100000 halo particles. I followed their orbits during 40 bar rotation periods and calculated their basic frequencies, namely the angular frequency $\Omega$, the epicyclic frequency $\kappa$ and the vertical frequency $\kappa_{z}$. For the two latter ones I used a spectral analysis technique (Binney \& Spergel 1982, Laskar 1990). In most cases there were several secondary peaks, and the frequency was determined by the main one. The angular frequency proved more difficult to calculate reliably, so I supplemented the spectral analysis with other, more straightforward methods, based on following the angle as a function of time. Agreement between the values for the angular frequency obtained by the various methods was found to be satisfactory for $85 \%$ or more of the particles.

An orbit is resonant if there are three integers $l, m$ and $n$ such that $l \kappa+m \Omega+n \kappa_{z}=m \Omega_{p}$, where $\Omega_{p}$ is the pattern speed of the bar. I will here restrict myself to radial (planar) resonances, for which $n=0$. The most important such resonances are the inner Lindblad resonance (ILR), where $l=-1$ and $m=2$, corotation resonance (CR), where $l=0$, and the outer Lindblad resonance (OLR), where $l=1$ and $m=2$. At CR the particles have the same angular frequency as the bar, while at the other resonances they make $m$ radial oscillations in the time they make $l$ revolutions around the center of the galaxy. Fig. 2 shows the number of particles (orbits), $N_{R}$, that have a frequency ratio $R=\left(\Omega-\Omega_{p}\right) / \kappa$ within a bin of a given width centered on a value of this ratio, plotted as a function of $R$.

Let me first describe the results for the disc components of MD and MH. The distribution in both cases is far from homogeneous, with strong peaks at the location of the main resonances. The highest peak, both for the MH and the MD disc, is for $\left(\Omega-\Omega_{p}\right) / \kappa=0.5$, i.e. at the ILR. Indeed, orbits making two radial oscillations in the time they make one revolution around the center of the galaxy are the backbone of the bar. In principle $x_{2}$ type orbits (cf. Contopoulos \& Grosbøl 1989 for a review) could also be found in this peak. We have, however, verified that the vast majority of the orbits here are $x_{1}$ type. The ILR peak in the $\mathrm{MH}$ case is roughly 1.5 times higher than in MD, consistent with the fact that the bar in the MH case is stronger. Model MD has a sizeable CR peak and a much lower one at the OLR, while model MH has only a small CR peak and no OLR one. The ratio of the height of the CR peak to that of the ILR peak is roughly 0.6 for the MD case, while for model MH it is only 0.09 . These differences are, to a large extent, due to the differences in the corotation radii of the two models. Thus at time 800 the $\mathrm{CR}$ radius is 7.1 for model $\mathrm{MH}$ and 4.6 for model MD. Similar differences are found for the OLR radii of the two models. Therefore these two resonances are in the outer parts of the $\mathrm{MH}$ disc and can trap only few particles. 
This is not the case for model MD, and the differences in the trappings are reflected in the differences in the heights of the respective resonant peaks. Model $\mathrm{MH}$ has also a clear peak at the inner 1:3 resonance and perhaps one at the 1:4. Model MH has a clear inner 1:4 peak.

The big surprise, however, comes from the halo component. So far considered as non-, or little, responsive, it shows, on the contrary, unmistakable signs of strong resonances with the bar. For both models the strongest resonance is CR, which proves to be much stronger in the halo than in the disc component. In fact the $\mathrm{CR}$ peak in the halo of model $\mathrm{MH}$ is higher than any of the disc peaks in MD. There are also sizeable peaks at the OLR.

There are important differences between the orbital structures of the MH and MD haloes, as was the case for the corresponding discs. The ILR peak of model MH is relatively high - the second highest peak for this model and time - and, in general, there is considerably more material between ILR and CR than in model MD, while the CR peak is $40 \%$ higher than in model MD. All these are in agreement with the fact that the $\mathrm{MH}$ halo is much more concentrated than the MD one, while the $\mathrm{MH}$ corotation is at a larger radius. For smaller values of $R$ the situation shifts and the peaks are stronger for the MD case. The OLR peak of model MD is more than 1.8 times as high as the $\mathrm{MH}$ one, and the $-1: 1$ peak is also clear in $\mathrm{MD}$, while for model $\mathrm{MH}$ this resonance and its surroundings have been depleted.

The relative and absolute heights of the resonance peaks vary with time. However, a large fraction of the orbits which are within a resonance peak at a given time continue to be so at a later time. Thus, comparing times 500 and 560, I find that 60 to 80 per cent of all orbits in one of the main resonant peaks at time 500 are found in the same peak at time 560. Similar numbers can be found by comparing times 800 and 860, except for the $\mathrm{CR}$ peak of the halo in the $\mathrm{MH}$ simulation, where the fraction falls to 55 per cent. Seen the uncertainties in delimiting the extent of a frequency peak, and the uncertainties in the

calculation of the frequencies, these numbers are compatible with a large trapping of particles in the resonances. A more detailed description of these changes, together with a discussion on their effect on the structure and the dynamical evolution of the galaxy will be given elsewhere.

\section{Discussion}

In the above I compared three simulations starting off with identical discs, but different halo components. Any differences in their dynamical evolution should thus be attributed to the haloes. The strongest bar forms in the most halo-dominated case, provided this is live, while the simulation with an identical but rigid halo forms only a mild oval distortion, in 
the inner parts only. This very big difference can be attributed only to the responsiveness of the halo. The disc-dominated case, i.e. the case with the less important halo component, formed an intermediate bar. I thus reach the interesting conclusion that haloes can, at least in some cases, stimulate the bar instability and lead to stronger bars.

Lynden-Bell \& Kalnajs (1972) have shown that disc stars at resonances can absorb or emit angular momentum, thus driving the dynamical evolution. Halo stars in resonance can also exchange energy and angular momentum (Tremaine \& Weinberg 1984). In general, the halo resonances will absorb angular momentum. Prompted by such considerations I searched for resonant stars in the halo, and found large numbers (at least after the bar has grown). Since these can exchange energy and angular momentum with disc resonant stars, they can stimulate the bar instability, contrary to previous beliefs, and thus explain why stronger bars can grow in more halo-dominated surroundings. The situation is particularly clear in the case of model MH, where, as Fig. 2 shows, there are hardly any absorbers in the disc to take the angular momentum emitted by the stars at the ILR. This task is thus necessarily performed by the halo resonant stars.

Since the bar is inside corotation, it has negative energy and angular momentum (e.g. Lynden-Bell \& Kalnajs 1972), and if it emits angular momentum it will be destabilised, i.e. it will in general grow stronger. This is in good agreement with the results on angular momentum transfer discussed at the end of $\S 2$. These show that the halo takes positive angular momentum from the disc/bar component and, since the disc, the bar and the final halo component all rotate in the same direction, this will destabilise the bar.

In the initial phases of the evolution the bar grows faster in the disc dominated surroundings, in good agreement with previous results (e.g. Athanassoula \& Sellwood 1986). However, in fine, the bar in the $\mathrm{MH}$ model reaches a higher amplitude, due to the stronger exchange of energy and angular momentum with the resonant halo stars. Thus a massive halo may help the bar grow and become very strong, so that very strong bars may be found in initially halo-dominated galaxies. In other words there is no disagreement between the results of e.g. Athanassoula \& Sellwood (1986) and those presented here or in AM or in Athanassoula (2002a, b).

Hernquist \& Weinberg (1992) checked the angular momentum given to a live halo by a rigid bar turned first on and then off adiabatically. In spite of considerable noise, they found indications that the angular momentum was deposited primarily at the resonances and that it is mainly absorbed. This tentative result is clearly established here, where both the disc and the halo are live, the evolution is not artificially forced and the resonances are clearly outlined. 
In the linear theory the exchange of energy and angular momentum is linked with the growth of the wave. In the nonlinear theory on the other hand it has been linked to the slowdown of the bar (Tremaine \& Weinberg 1984, Weinberg 1985, Hernquist \& Weinberg 1992). Both effects are clearly visible in the simulations presented here and should be physically linked.

The orbital structures revealed in Fig. 2 are not specific to the models discussed above. I have repeated a similar analysis for other times and other simulations and found similar behaviours. They should thus be representative of a wide class of models.

I am thus proposing a new instability mechanism, by which the halo will stimulate, rather than restrain, bar growth. This of course will only work if the halo is non-rigid and is capable of absorbing positive angular momentum. Similarly the bar should also be non-rigid. Further analysis of this mechanism will be given elsewhere.

I would like to thank A. Bosma, M. Tagger and F. Masset for stimulating discussions, and A. Misiriotis and J. C. Lambert for their help with the software calculating the orbital frequencies. I would also like to thank the Region PACA, the INSU/CNRS, the University of Aix-Marseille I and the IGRAP for funds to develop the GRAPE computing facilities used for the simulations discussed in this paper.

\section{REFERENCES}

Athanassoula, E. 1984, Physics Rep., 114, 319

Athanassoula, E. 1996, in IAU Colloq. 157, Barred Galaxies, ed. R. Buta, D. A. Crocker \& B. G. Elmegreen (San Francisco ASP), 309

Athanassoula, E. 2002a, in IAU Symp. 208, Astrophysical Supercomputing Using Particle Simulations, eds. J. Makino \& P. Hut, PASP, in press, and astro-ph/0112076

Athanassoula, E. 2002b, in ASP Conf. Ser., The dynamics, structure and history of galaxies, eds. G. S. Da Costa \& E. M. Sadler, in press and astro-ph/0112077

Athanassoula, E., \& Misiriotis, A. 2002, MNRAS, 330, 35 (AM)

Athanassoula, E., \& Sellwood, J. A. 1986, MNRAS, 221, 213

Binney, J., \& Spergel, D. 1982, ApJ, 252, 308

Combes, F., Debbasch, F., Friedli, D., \& Pfenniger, D. 1990, A\&A, 233, 82 
Contopoulos G., Grosbøl P., 1989, A\&A Rev., 1,261

Debattista V. P., \& Sellwood, J. A. 1998, ApJ, 493, L5

Debattista V. P., \& Sellwood, J. A. 2000, ApJ, 543, 704

Hernquist L., 1993, ApJS, 86, 389

Hernquist L., \& Weinberg. M. D. 1992, ApJ, 400, 80

Laskar, J. 1990, Icarus, 88, 266

Little, B. \& Carlberg, R. G. 1991, MNRAS, 250, 161

Lynden-Bell, D., \& Kalnajs, A. J. 1972, MNRAS, 157, 1

Ostriker, J. P., \& Peebles, P. J. E. 1973, ApJ, 186, 467

Sellwood, J. A. \& Wilkinson, A. 1993, Rep. Prog. Phys., 56, 173

Tremaine, S., \& Weinberg, M. D. 1984, MNRAS, 209, 729

Weinberg, M. D. 1985, MNRAS, 213, 451 

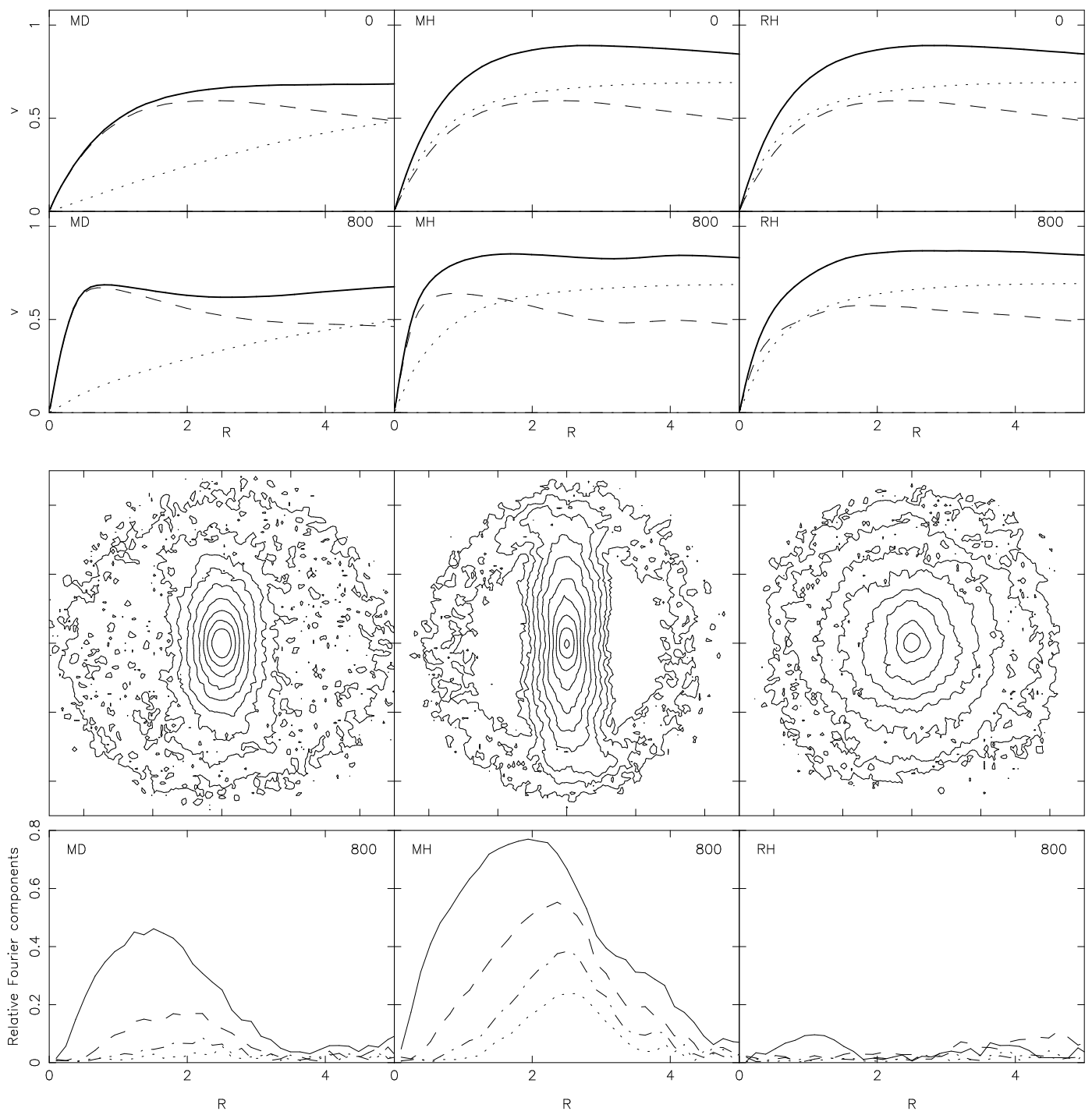

Fig. 1.- Basic results for the three simulations discussed in the text. The left panels correspond to model MD, the middle ones to model $\mathrm{MH}$ and the right ones to model $\mathrm{RH}$. The first and second row of panels give the circular velocity curves at $t=0$ and 800 , respectively (solid lines). The contribution of the disc component is given by a dashed line, and that of the halo by a dotted line. The third row gives, again for $t=800$, the isodensities of the disc component when seen face-on. The size of the square box for the third row of panels is 10 initial disc scale lengths. The last row gives the relative amplitude of the $m=2$ (solid line), $m=4$ (dashed line), 6 (dot-dashed line) and 8 (dotted line) components of the mass, again at $t=800$. 


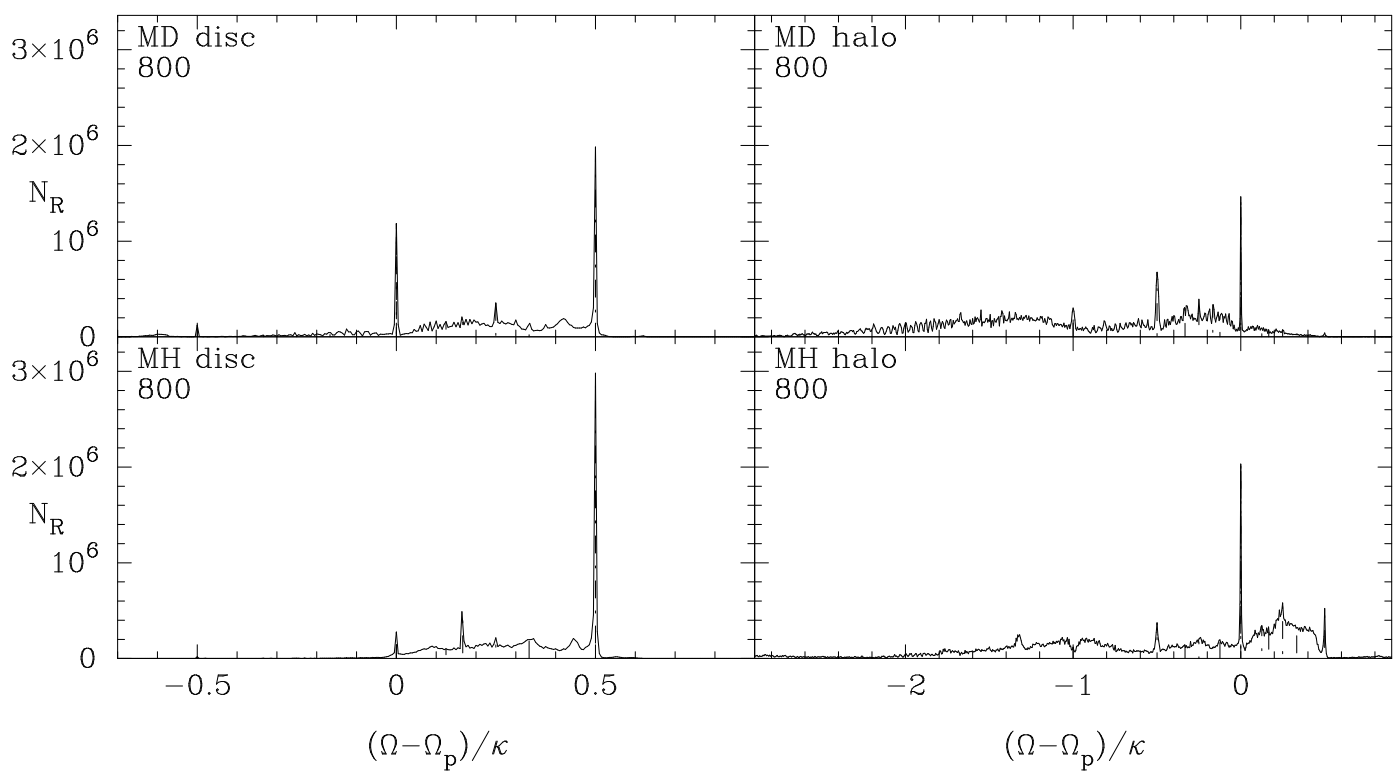

Fig. 2.- Number density, $N_{R}$, of particles as a function of the frequency ratio $R=(\Omega-$ $\left.\Omega_{p}\right) / \kappa$, for simulation MD (upper panels) and MH (lower panels) at $t=800$. The left panels correspond to the disc component and the right ones to the halo. The dot-dashed vertical lines give the positions of the main resonances. The results for the halo component have been rescaled, so as to take into account the different number of particles in the disc and halo components, and thus allow immediate comparisons. 